\title{
Managing peer support workers in Swedish mental health services - a leadership perspective on implementation and sustainability
}

Katarina Grim ( $\boldsymbol{\sim}$ katarina.grim@kau.se )

Karlstad University

Magnus Bergmark

Umeå University

Elisabeth Argentzell

Lund University

David Rosenberg

Umeå University

\section{Research Article}

Keywords: peer support, manager perspectives, sustainable implementation, mental health services

Posted Date: June 1st, 2022

DOI: https://doi.org/10.21203/rs.3.rs-1300247/v2

License: (9) This work is licensed under a Creative Commons Attribution 4.0 International License. Read Full License 


\section{Abstract}

Even though it has been demonstrated that peer support contributes to positive outcomes for service users, organizational implementation issues remain. The aim of the current study was to examine an implementation of peer support from the perspective of managers in order to develop knowledge of factors influencing sustainability of this initiative.

Eighteen managers were interviewed in connection with the introduction of peer support in sixteen mental health settings. The interviews were analyzed utilizing inductive as well as deductive approaches where, in the latter, a theoretical framework was applied to identify critical implementation components at the levels of organization, team and continuous support.

The results suggested that managers were predominantly positive in their evaluation of peer support as a recovery-oriented and user-friendly addition to their services, but noted developmental issues regarding role, professional identity, supervision and financing in relation to other traditional personnel. The involvement of the user movement, especially with regard to training and supervision helped prepare staff and support peer workers, yet there was some apprehension attached to the critical scrutiny that this 'outsider' perspective might imply.

The results confirm previously noted uncertainties regarding peer support as an integrated component of mental health systems. While offering clues as to the factors that would further define and integrate peer support, this study also highlights a number of challenges regarding role, identity and connection to the user movement, that need to be considered by managers before assuming that peer support should be implemented with the same approach as other interventions.

\section{Introduction}

Peer support involves people with lived experience of mental health problems who are trained and employed to offer support to others using mental health services. A peer support worker (PSW) can act as a motivational role model and can promote hopefulness by sharing their experiences with mental health problems, psychiatric care, support and recovery (Repper \& Carter, 2011; Rosenberg \& Argentzell, 2018; Viking et al., 2022). The majority of studies describing peer support have been positive and qualitatively described the beneficial, experiential aspects of clients meeting peer support workers. However peer support has also been shown to lead to positive outcomes in the form of higher quality of life and functioning (Chinman et al., 2014; Fuhr et al., 2014; Rogers et al., 2016), recovery and empowerment (Farkas \& Boevink, 2018; Salzer et al., 2016).

At the same time the introduction of peer support seems to challenge traditional, medically oriented mental health organizational cultures to become increasingly recovery-oriented and focused on user participation (Davidson \& Guy, 2012). In fact, introducing a PSW has been viewed as the foremost step towards establishing recovery-oriented care (Slade et al., 2014; Slade et al., 2017). However, although peer support has existed since the 90ties, there remain concerns about the organization and implementation 
of peer support. Implementing peer support has been noted to be a complex process that needs to involve service users, managers and staff and the PSWs themselves (Davidson \& Guy, 2012; Farkas \& Boevink, 2018). The importance of clearly defining the PSWs' tasks has been identified, at the same time as flexibility is needed for developing the PSW role based on the specific needs of the service (Crane et al., 2016; Silver \& Nemec, 2016; Viking et al., 2022). Implementation studies additionally highlight difficulties associated with integrating peer support in existing practice and achieving sustainable implementation (Ibrahim et al., 2020; Kent, 2019). A recent review of implementation barriers identified a range of challenges, including cultural impediments, poor organizational arrangements, discrepancies regarding roles, and inadequate predominating mental health policies (Ibrahim et al., 2020). The centrality of manager commitment is specifically underscored in studies on peer support as well as in the implementation literature (Aarons et al., 2011; Damschroder et al., 2009; Ibrahim et al., 2020).

Although peer support is well established internationally, it has only recently been introduced in Sweden. In order to introduce a clear PSW role within Swedish mental health care, a Swedish model for implementing peer support was developed by the national user organization NSPH (National Collaboration for Mental Health). In this model, the user movement, in the form of NSPH, recruits, trains, supervises and coordinates the implementation in the services. The model consists of a comprehensive recruitment and training program for the PSWs, extensive information in the form of manuals and brochures, staff training as well as frameworks for supervision and implementation support. The model is based on internationally established knowledge on peer support, knowledge drawn from research as well as collaboration with international user organizations (Kemp \& Henderson, 2012; Repper et al., 2013) and have been formed to be suitable for implementation in a Swedish mental health context. The Swedish PSW model provides a unique organizational structure, differing from those developed in many other countries, in that the PSWs are initially employed through the user organization (NSPH) (Nordén \& Falkman, 2018). This structural connection is intended to ensure that the PSW has a strong association to the principles and aims of the user organization.

Since the introduction of peer support in Sweden is at an early stage, research is scarce within a Swedish context and studies on implementation are lacking. However, early findings reporting experiences of introducing peer support are in line with international research and suggest positive outcomes on a user level (Rosenberg \& Argentzell, 2018). Even if evidence for specific effects is still scarce, peer support is currently classified as a rehabilitation strategy that is important for recovery and is presented as a promising research and development area in Swedish mental health care (Socialstyrelsen [National Board of Health and Welfare], 2017).

In view of the centrality of leadership engagement for sustainable implementation of peer support (Damschroder et al., 2009; Ibrahim et al., 2020), insight into managers' experiences and views of implementation processes can provide guidance in tailoring future implementation strategies.

\subsection{Research aims}


While peer support has a long history within mental health care and with positive outcomes on a user level, implementation issues remain, especially in relation to sustainability over time. The current study has therefore focused on the implementation of peer support as an innovation in Swedish mental health care by following and exploring a structured user movement initiative. Using an implementation framework, the study aims to examine managers' perspectives of the implementation process at the included sites and what specific issues need to be considered in order to facilitate the sustainable implementation of peer support in mental health services in the future.

\section{Methods}

\subsection{Study Design}

The study has a naturalistic approach, following a systematic rollout of a national peer support project in three regions. A multiple case study design was used, where each case, individual and collective, was instrumental to answering our research questions (Hollweck, 2015; Stake, 1995). By such an approach, a multitude of context-specific dynamics and interrelationships may be captured and analyzed (Erlandson et al., 1993).

Since the aim of the study was to explore implementation processes as they played out in real life settings, without 'interference' or control of the researchers, interviews conducted in connection with the start-up of the projects ranged from 3 - 6 months after the initial employment of a PSW. This design gave us the opportunity to study implementation components critical when designing implementation plans (e.g., prior knowledge, commitment and incentives), and those that are critical as the implementation progresses (e.g. role distribution, technical and administrative support).

In harmony with recent research highlighting the importance of applying an implementation framework to recognize best practice for supporting implementation (Mutschler et al., 2021), the current study utilizes a framework offered by the Sustainable implementation Scale (SIS) (Markström et al., 2018), to provide an organizing structure in data collection and analysis of results. SIS is a framework for discerning factors that may hinder or facilitate successful implementation.

\subsection{Study Context}

NSPH is an umbrella organization consisting of the country's largest service user associations in the field of mental health. NSPH became aware of and began to study peer support as provided by international organizations and collected reports and conducted site visits to a number of peer support projects operating in other countries (Repper et al., 2013).

The current study is conducted within the scope of a larger, three-year multi-phase project aiming at introducing peer support in the context of the Swedish mental healthcare system. The project was launched by NSPH in March 2016 and was financed by the Swedish Inheritance Fund. Before launching 
the project, NSPH established collaboration with researchers within the national research network CEPI (Center for Evidence-Based Psychosocial Interventions) who developed a model for evaluating the implementation of peer support based on international knowledge about the implementation of evidencebased methods.

The sites that were followed corresponded with the rollout process. Local municipalities and regional psychiatric services also needed to express interest and willingness to collaborate with researchers as a part of their evaluation and follow-up locally. In Sweden, there are two primary providers of mental health services, those based in the municipal social services offering residential support, case management and occupational therapy and region-based in- and outpatient psychiatry offering medical treatment and psychotherapy. In the current study varied services from both these actors are included, see table 1 . Within the context of the project, a variety of qualitative data have been collected to capture the perspectives of various stakeholders, e.g. staff, PSW:s and service users. A study on service users' experiences of having contact with PSW:s has been published, and in this current study the focus is on the managers' views of the implementation (Rosenberg \& Argentzell, 2018).

Please insert table 1 about here

\subsection{Data collection}

All of the included managers were suggested by our contacts at the participating sites as those who had most operative responsibility for the implementation and all agreed to be interviewed. In two cases, two managers were identified and interviewed for the same service.

Interviewers, who were either researchers or persons with service user experience interested in research, were trained, closely supervised and guided by $X X$ and $X X$ in order to secure the quality of the project. The same interview protocol was utilized in all interviews. Individual Interviews were conducted with 18 managers and were recorded and transcribed verbatim.

Knowledge from the field of implementation research was captured by using the Sustainable Implementation Scale (SIS) (Markström et al., 2018). SIS consists of 24 components sorted in the categories organizational level, team level and continuous support (see table 2), all of them critical for successful implementation according to well-established research reviews from the field of implementation science (Damschroder et al., 2009; Durlak \& DuPre, 2008; Fixsen et al., 2009; Meyers et al., 2012). The implementation components were utilized as deductively derived codes to design the interview protocols and to sort the data.

Please insert table 2 about here

\subsection{Data analysis}


All data were analyzed using qualitative content analysis (Hsieh \& Shannon, 2005). The data was approached from a directed implementation perspective in which implementation components in the SIS provided orienteering concepts to develop relevant issues, processes and interpretations in order to identify and cluster initial codes. After these steps, an abductive content analysis incorporating both inductive and deductive approaches in the different stages of the analysis was performed (Graneheim et al., 2017). At a next stage, a more unconstrained matrix was developed where theoretical concepts guided the discovery of categories, rather than steered them directly (Erlingsson \& Brysiewicz, 2017).

All four authors participated in the data analysis process to provide multiple viewpoints, ensure consensus and strengthen trustworthiness of the analysis. The authors contributed with diverse knowledge perspectives from within the field of mental health; academic and clinical practice from occupational therapy $(X X)$, social work (XX and XX) and service user (XX).

\section{Results}

In the analysis, three principal categories with related subcategories were identified. Additionally, an overarching theme running throughout the categories was distinguished and will be discussed below. The findings suggest that managers find themselves in a quite undefined field, where they attempt to develop their perspective on peer support and justify decisions regarding their inclusion in their organization, without clear boundaries or structures for doing this. We describe this theme as Navigating liminal spaces when implementing peer support in mental health systems. Liminality denotes transitional or transformative states, where usual practices and structures have been suspended but where new ones have not yet been established (Czarniawska \& Mazza, 2003), and has been applied in recent literature to explore and describe the complexities involved in transformational change in roles and cultures within mental health systems (Agrawal et al., 2021).

The theme, the main categories and subcategories are presented in table 3. Along with the descriptions of the categories below, citations are given where references are to the managers in regions $A, B$ and $C$, (as listed in table 1), and are identified as follows: (A1-7), (B8-12) and (C13-18).

\section{Please insert table 3 about here}

\subsection{Incentives and conditions}

This main category includes the managers' descriptions of factors that had contributed to, or otherwise affected the decision to commit to implementing peer support in their services.

\section{Previous Experience and Commitment}

Amongst the managers, the initial enthusiasm for peer support implementation ranged from being a bit hesitant to being an engaged champion. Those who were hesitant had little prior knowledge of peer support and typically had no other incentive to join the project other than that of saying yes to something "exciting and interesting to test" (C14). 
On the other end of the continuum, several managers expressed a serious dedication to the implementation of peer support. These managers typically had theoretical as well as experiential knowledge of recovery-based practice and user involvement. Some of them had been pioneers in peer support implementation in Sweden and had also often been the initiators and champions for joining the current project.

In describing what had contributed to the decision to take on a PSW, they frequently expressed a commitment to strengthen user participation in the services. Some noted how this drive had been spurred, during the last decade, by the increased emphasis in directives and policy on user participation. The activities of NSPH and a national governmental initiative focusing on psychiatry were noted as important elements in supporting this shift in the caregiving culture. Some managers described the current project as providing an opportunity for taking the next step, and when offered the opportunity to employ a PSW it was "natural to say yes" (A5).

\section{Opportunities for Full-Scale Support}

It was generally appreciated that the "all-inclusive concept" (A5) implied no financial and little administrative burden on the services. Some managers had experience of peer support within time-limited projects where uncertainties regarding the position had led to problems. The fact that the current project involved an 'implementation package' including staff education, supervision and facilitation, contributed to their enthusiasm to be involved. Other managers described how witnessing other services successfully implementing peer support had stimulated their willingness to try, some having waited for such an opportunity.

Many described the full external funding as a major incentive for joining the project, where they had the opportunity to try out something they really wanted despite limited financial budgets:

The budget allotments that we have to follow aren't always in line with our real needs. So, I considered this a golden opportunity (A7).

The most committed expressed regret that the only opportunity they had for taking on a PSW was within the framework of a time-limited project rather than a proper, internally determined and financed implementation.

\section{User Movement Collaboration}

The category user movement collaboration describes comments on various forms of networking with user organizations locally as well as nationally that had affected the conditions for implementation.

Among the managers, there was a wide variety of opinions as to what extent a collaborative culture had been established with user organizations, and regarding the quality of the implementation support from NSPH. Many noted how regions who had established working collaborations with the user movement 
could contribute to wider progress through networking. This development was described as "spreading like ripples on water" (B12).

Regarding user organization involvement in the implementation process NSPH was clearly the user organization who had the primary role, although one region (C) had developed its own local structure for supporting user influence. One manager noted how the focus on user involvement in general had been instigated by national initiatives and directives from the central authorities, and how NSPH now had moved forward with this mission in a commendable way:

Then it is not least NSPH who has taken the baton and raised the issues nationally, and when it comes to PSW they have developed training packages, structure and organization of implementation and received a very positive and well-earned response. By this, PSW had had a real boost in recent years (B11).

The collaboration with NSPH before entering the project was commonly described as supportive and well structured. Not least, managers reported how the presence of NSPH in meetings had provided an important forum for ventilating both doubts and opportunities that engaging a PSW might entail. However, one of the managers noted a lack of support from local user organizations. The service was located in a rural area and they described the national umbrella organization as having overshadowed a focus on involving local user organizations.

\subsection{Implementation and Organization}

In this main category, various factors are described that were viewed as significant for the implementation and organization of peer support in the service.

\section{Preparation Phase at the Start}

This category describes issues that affected staff readiness for taking on a PSW. It concerns the managers' descriptions and views of staff training, of staff attitudes and concerns, and of their own actions in the introduction of a PSW in the team.

The managers often underscored the importance of anchoring new interventions in the team, of the team having the opportunities to ventilate questions, concerns and opinions in open discussions. It was noted how introducing a new profession constituted a challenge for the team, something which needed to be handled with sensitivity.

Managers described an initial mix of attitudes in the working team, from those who showed great enthusiasm to those who were entirely negative. Amongst those with the most skeptical staff members there was one that even had been worried that a couple of staff would treat the PSW badly. Typically, teams who had previous experiences of peer support or of other models for user involvement were relatively positive to taking on a PSW. According to some, they "had made the journey" (C18), were "one step ahead in the thinking" (C13) and were "further along" (B12) than many other services. 
Managers expressed that staff training had been much appreciated and experienced these as having contributed to readiness. However, the degrees to which staff had participated in training varied.

\section{The Working Role of the PSW}

The category the working role of the PSW concerns issues of developing and defining the role. It involves managers' experiences of initial concerns amongst staff relating to role expectations and identity issues. In addition, it concerns the practical, administrative aspects of the role. In view of the SIS scale, these matters might be understood to concern implementation components of organizational fit of the intervention.

Defining the role was viewed as important but also difficult since all involved; staff, managers and the PSW, had to "deal with grey zones" in relation to the traditional clear division between staff and service users.

The managers reported that some of the initial concerns expressed were that the PSW would take over many desirable but informal contacts with the patients or disturb the "free zone" (C16) in the personnel room where they could "breath out and ventilate" (C16). They expressed their own uncertainties about what the PSW would be allowed to "see and hear" (C17), and worried that the PSW, who lacked care training and might be particularly vulnerable and have difficulties setting appropriate boundaries.

Several managers reported staff skepticism regarding having "a staff member that is simultaneously really a service user" (A3). Notably, the prospect of taking on a PSW was described as emotionally charged among staff and something that was commonly thought to require overcoming one's "prejudices" (B12, C15) and "fears" (B12). Such concerns were often noted to be counteracted by receiving clear descriptions of roles and responsibilities during the training. In some cases, the managers reported that they themselves had decided on work tasks for the PSW while others had allowed the PSW role to unfold or to develop in practice. Some appreciated the excitement of "breaking new ground" (C18) and the necessity to hold back so as to allow for the fresh and different perspectives of the PSW to discover what needed to be added to the routine practice. As one manager observed:

One of the benefits of peer support is to find the gaps that we have not noticed, therefore we have said that we will take it easy (C18).

Perceptions of the organizational belonging of the PSW sometimes reflected an uncertainty and ambiguity. The managers in the region where the PSW were employed within the service generally expressed how this was the natural and optimal arrangement in order for PSW to be "for real" (B12), ensuring that the PSW becomes a valued member of the team. These managers describe how the PSW participated in staff meetings and trainings on the same conditions as other staff, "like one of us" (C15, C17).

Managers expressed the difficulties involved in handling this unfamiliar working role, not knowing whether to give special consideration to the PSWs due to their potential vulnerability or whether to "treat 
them as anyone else" (C15).

They expressed a variety of expectations regarding the PSW role, including "a role that is supposed to develop the service" (C6), and the PSW as someone who" helps build alliances" (B9) and "supports communication" between users and staff (B11). The managers describe the PSW as "serving as a link [to the users]" (A2, B9, C15) or "listening with a third ear, on another frequency ... and then conveying it to the staff" (A6). When describing expectations for the PSW role as differing from traditional roles, managers described how the PSW should be prone to "see health factors and strengths" (B12), to sit down and talk with clients "without having an agenda" (B2), and how the PSW may instill hope by being "living proof" (B9) that recovery is possible.

A characteristic that was frequently mentioned as desirable in a PSW was that the person should be "far along" (B12, C15) in or even having completed his or her journey of recovery. This is expressed as important in that it ensures that the PSW has a "certain maturity" (A7) and "can tell the difference between what is professional and unprofessional" (A5) and "what is private and personal" (C15). The person needs to be "strong and stable enough" (A7) and "in control of his or her baggage" (12B) not to "take too much room for him-/herself and start things he or she cannot handle" (A7). They even described having come "a long way in their recovery" (C15) as insuring that the PSW is "not there for his or her own sake" (A2, C15) in order to "process experiences and past events" (C15) and that the PSW does not "carry any resentment" (C15).

The specific tasks of the PSW, as described by the managers, involved participating in group activities, "being out on the floor" (B12), being available for spontaneous talks, "for taking that walk and for staying a bit longer in a conversation" (C18) than is possible for ordinary staff to do. Many of the managers suggested that the PSW should not be involved in documentation and treatment, while others, including some of the most experienced 'local champions' stated that the PSW should have full access to journals and participate in treatment conferences.

\section{Continuous Support}

Throughout, implementation was described as more successful when continual support was provided by $\mathrm{NSPH} \mathrm{n}$ the form of facilitation, guidance and supervision for mangers, staff and for the PSWs. In many cases managers expressed that NSPH had been "present and accessible" (B11) for support in any issues that arose during the project period while some others had experienced such support as largely lacking.

Managers reported that external supervision was provided for the PSW, most often from NSPH. Generally, they appreciated this form for external supervision, expressing the value for the PSW to receive collegial support and guidance under the auspices of the user organizations. One manager emphasized how substantial support is needed for the PSW to handle a challenging role, and to be supported as a profession: 
I think it is extremely important that they are reinforced as a professional group, and therefore receive external guidance together (A6).

However, in one case, no supervision had been provided, causing an extra burden on the supervisor who had had to spend "more talking time with the PSW than with any other staff" (A5).

\subsection{Experiences of Peer Support}

This main category involves the managers' experiences and perceptions of different aspects of the implementation process: the PSW in the client work, in the working team and the design of the project organizations.

\section{The PSW in the Client Work}

The category the PSW in the client work includes managers' descriptions of positive as well as problematic aspects of the PSWs contacts with service users. In view of the SIS framework (table 2) this category can be understood to concern issues of organizational fit as well as experience of the model.

Typically, managers stated that they had limited knowledge of specific service user experiences of the PSW. Still, they typically reported having received positive feedback from staff members, reporting that the PSW were in demand, that service users appreciated "speaking to someone who understands" (C18). It was noted how the PSW conveyed to the service users "hope by their mere presence" (B12) how "illness experiences [may be] a resource for oneself as well as for others" (C18) and that "it is possible to make something positive out of the difficult" (B8). Initial fears among staff that there would be much "illness talk" (A1) in the conversations had proved unfounded. Instead it was noted how the holistic perspective of the PSW gave them a stronger focus on "wellness factors" (B12) than other staff.

In many cases, the mangers noted how they were surprised at the ability and competence of the PSW:

I did not have very high expectations..., I was probably set on that this is a person who has a chronic psychiatric illness and that it was actually not so long since he was ill and required inpatient care... So I actually did not think he had the level he actually has (B8).

It was noted that the PSW had been able to earn the trust of service users in ways that no other staff member had and that meetings with the PSW had been requested by service users who had never asked for any other staff. Some reported how ordinary staff, on several occasions, had not succeeded in reaching certain individuals, but how the PSW who had posed a question from another perspective had received an immediate response. By some, it was also noted how the "other perspective" (B12) of the PSWs is important to bring to decision-making processes regarding the care and support of service users. In addition, it was noted how the shared experience enabled the PSW "to be braver" (C18) than ordinary staff and to "tiptoe less" (C18) around difficult subjects. Lack of courage was observed to constitute an "Achilles heel" (C18) for professionals who tend to avoid such subjects for fear of making things worse. 
However, at the same time that managers highlight the alliance building power of sharing personal experiences and of daring to talk about the most difficult matters, the importance of the PSW setting boundaries "between personal and private" $(\mathrm{C} 15, \mathrm{C} 18, \mathrm{~A} 4, \mathrm{~A} 6)$ was commonly underscored as vital and experienced as a major challenge. Some staff expressed concerns to the manger regarding the support provided by the PSW, for example having provided "too much support" (C15).

\section{The PSW in the Working Team}

The category the PSW in the workplace involves the managers' descriptions of their experiences of having the PSW in the service in relation to issues of working climate, staff attitudes, service provision and organization. In relation to the SIS framework, experience of an intervention provides an organizational level implementation component determining readiness for further implementation.

Some managers reported that staff who were initially skeptical to peer support were still cautious in their judgement. It was noted by one of the experienced managers that it is important "always to keep the demarcation alive between what staff does and what the PSW does in order to avoid a competitive situation" (B12). It was observed that a PSW could build deeper relationships with service users. One manager reported how this creates tension that has "led staff to denigrate the PSW for being too unrestrained" when they ought to have welcomed this "knowledge contribution in the service" (B12). Correspondingly, "finding the role" and seeing to that the PSW does not "take over the tasks of other staff members" (C16) was described as a major challenge, one that was part of the particular focus for responsible managers.

When talking about experiences from the peer support project, managers often highlighted positive, personal characteristics of the PSW. In a few cases, personal characteristics of the PSW were described as problematic, and often those traits were related to the mental health problem of the PSW such as being "too emotional" (A5) or having "difficulties with change" (A3).

Overwhelmingly however, managers gave voice to positive experiences and in many cases, having a PSW in the service had far exceeded their expectations. Initial fears that the PSW would disturb the conversational climate in the team had proved unfounded. Typically, the PSW had come into the team without difficulties and had eased the burden of staff as well as managers.

It was noted how the PSWs had supported user involvement in the services, giving guidance on how to think and work and contributing ideas and supporting initiatives that would support self-help and recovery. Many managers express how the PSW has provided the staff with new perspectives and generated shifts in staff attitudes. One manager noted how the jargon was affected and that the presence of the PSW "makes it harder to talk in derogatory terms about the service users" (C13). In many cases, the managers described how staff actively consulted the PSW and received guidance on difficult matters regarding specific service users. In the following quote, a variety of positive effects and contributions are expressed: 
Peer support changes attitudes and the whole atmosphere at the units. For example, staff express that at treatment conferences they twist and turn the perspectives a little more, they problematize a little more and they actively demand a PSWs' point of view. The PSW is seen as an important source of knowledge, that is important to highlight. They provide new ideas and ways of thinking. Several people have noticed that conversational tones and topics discussed in the staff room have also been enriched (B10).

Many managers gave concrete examples of knowledge provided by the PSW, specifying how the PSW had helped the staff understand how mental health problems could be experienced from the inside and raising their awareness on how to recognize and interpret signs of illness:

The PSW gave us a lot with his openness, a lot of information about how the disease goes, what conditions you might have, how we should for example interpret certain behaviors, certain situations for people. (A4).

Another example of this valued perspective was provided by the manager of a clinic who noted how the PSW had a holistic and "forward looking" (C18) perspective on service users' situation that is often lacking among staff who, on a daily basis, deal with people in acute crisis" C16). Managers often described the presence and perspectives of the PSW as "eye opening" (B10) and as "providing a totally new way of thinking" (B12). Some noted how the PSW provides a critical eye on the service and has spurred self-reflexivity among staff making them "question what they did earlier" (A6), sometimes by "expressing opinions that are not always comfortable" (A6). Throughout, such scrutiny was described in positive terms, as in the quote below:

It feels like a reinforcement and complement ... that our services in some way get better and we are even monitored and questioned in another way when he asks his questions and approaching things differently. It really adds something (A5).

Some managers remarked how staff often give themselves "interpretive prerogative" (C18) of service users' behaviors and tend to be "too solution focused" (B10) and sometimes over-caring in their eagerness to help, and that the PSW had helped them hold back from handing out solutions to service users' problems.

Moreover, it was noted by one manager that the PSW had made it easier for staff "to dare to open up" (B11) and talk about their own experiences of mental health problems by normalizing such conversation. Another manager held that ordinary staff should be more open with their own experiences in order to avoid a "too strong focus on [the PSW as an] individual person" (C16)

Some experienced the project as sufficiently structured and others that structure was lacking. As for practical and administrative issues, many expressed how they would have liked more guidance and regulation on issues such as employer responsibilities, wage determination and sick leave. Some experienced the short time span of the project as stressful. It was observed by one manager that "implementation work takes time" (B11) and by another that more time would be needed for them to "try 
properly" (A6). In light of the SIS framework, these views can be understood to reflect various levels of readiness and consequently of organizational fit.

\section{Thoughts on the Future}

The category thoughts on the future involves ideas for further implementation, on perceptions of barriers and prerequisites and lessons learned.

In most cases, managers expressed a wish for continuing with peer support. Some reported that at the end of the project, they felt ready to manage it on their own, while others expressed that they hoped for a continuation, citing a need for further support from NSPH. In these cases, they reported the time-limited project implied uncertainties and created stress, making it difficult for the managers to make plans and for the PSW to have control over their future. They expressed a vagueness about future opportunities that prohibited long-term and systematic planning:

In order for us to plan and for her to be part of our planning, I would like to know if we have a future together or if things are just sliding along haphazardly. (B11).

In many cases, managers expressed a desire to employ the PSW permanently, so that they had job security and that the position would be sustainable, however financial resources had not been determined. Many also reported facing major reductions and recruitment freezes, ruling out any such employments. Without an organizational decision to dedicate specific funds they feared that employment of a PSW would come at the expense of another member of staff. As one manager noted: "if we are to keep her, we have to remove someone else, and who then is going to go? (A6)"

However, amongst the managers with long experience and commitment, there was a perception that a PSW indeed could be hired instead of someone from another occupational category when a vacancy occurred, noting that service needs and goals have changed during the last decades.

These managers underscore the importance of setting aside funds centrally, for providing legitimacy and demonstrating that PSWs are valued as important members of teams:

It is not enough to just say that you are for something, giving a pat on the back that you are doing a good job, you also need some financial incentives to show that you are serious about this. (B10).

Further, another committed manager expresses that although participation in fully funded projects serves to "generate a need that makes it difficult to let the PSW go when the project ends ... it is now high time that these uncertain projects are transformed into regular employment "(B8).

The dynamic challenge that managers face in Navigating liminal spaces when implementing peer support in mental health services was the overarching theme as presented in table 3 . The theme was developed in response to the various "grey zones" that the categories included. These became evident when exploring expectations and definition of peer support work, where roles were unclear in relation to 
professional identity and to the practical structure of the position. The role and identity of the PSW can thus be described as inhabiting a 'liminal space' in that it exists in-between the role of the service user and the role of the professional. Similarly, it was apparent how this in-between existence and this inherent uncertainty was evident at all levels from the micro to macro, regarding instrumental (practical, administrative, organizational) issues as well as matters of knowledge legitimacy and cultural values. The implications for implementing peer support while navigating various ambiguous or transitional fields will be further elaborated below.

\section{Discussion}

The study presented here was part of a larger project following the implementation of peer support in Sweden, that was initiated and supported by the national user movement (NSPH), The aim of this study was to develop knowledge of implementation of peer support and identify specific issues that might need to be considered for future implementation attempts, as reported by program managers. In the following discussion, we will first comment on some of the general conclusions regarding implementation that might be drawn from the analysis of the interviews in relation to the implementation components that informed the interview protocol.

It became clear that the decision to introduce a PSW was only rarely based on a needs' analysis and that broad support at higher levels of the organizational leadership was largely lacking. The implementation was often prompted by external pressure from national and regional health care and social authorities and as a response to general directives for increased user participation. There was often little evidence of prior experience of peer support or other structured models for user participation in service provision, and the majority of reported experiences had been in the form of short-term projects.

The engagement of the managers interviewed varied, with some having experienced external pressure while in other cases, the respondent manager had been driving the implementation, playing the role of a local champion, while their superiors rarely showed such commitment. The overall attitude managers described suggested that managers at all levels, as well as local politicians, were curious and enthusiastic, open to change when opportunity was given, but rarely prepared to devote resources that would drive and support such a change over time. This phenomenon of lone champions initiating an implementation without full commitment from the surrounding organization and leadership is commonly highlighted in implementation literature as a risk factor that creates vulnerability (Bergmark et al., 2019). The results suggest that while the needs that PS might fulfill in the program were not investigated prior to implementation, the experience of implementing peer support contributed to understanding and defining the need for continued investment.

Consistent with previous research (Bergmark et al., 2019; Ibrahim et al., 2020; Rubin et al., 2016), limited funding was commonly noted as a problem. PSWs were typically underemployed in that they often did not have enough working hours and future financial arrangements were insecure. Even though the value 
of peer support generally seemed positively acknowledged by managers, it was evident that ideological acknowledgement of the value of peer support clashed with the reality of limited resources and budget.

The managers expressed a desire for clarity in role definition, task descriptions and administrative structures. At the same time however, and consistent with implementation literature (Damschroder et al., 2009; Durlak \& DuPre, 2008), the value of allowing for local adaptions was emphasized. These contrasting views indicate that there are advantages and disadvantages attached to different degrees of structure. Greater degrees of micro-management might curtail the space for the development of a new professional role and knowledge perspective.

The introduction of peer support was also dependent on the external user organization educating and introducing the PSW. While the PSWs themselves received consistent education and preparation, the results confirm that there was a great deal of variation in the training of staff. In accordance with the Quality Implementation Framework (Meyers et al., 2012), staff preparation seemed to increase readiness and reduce opposition to implementation. The results indicate that the need for continuous support is clear, but express ambivalence as to whether this should be provided by user organizations or internally, suggesting advantages and disadvantages for both models.

Examining the implementation in terms of organizational fit (Damschroder et al., 2009; Markström et al., 2018) it was evident that peer support does not only involve developing a function, one requiring structural factors to be in place, but also necessitates a cultural fit. It was evident that a cultural shift has begun and is ongoing but has not yet been achieved. Peer support was most often viewed as a welcome, additional resource and a promising complement to regular practice. Only rarely did the managers suggest that regular practice needed to be challenged in order to make room for PSWs. These diverging views might be understood to reflect the varying sense of the legitimacy of and commitment to peer support, as well as a concrete example of the uncertain nature of this form of support in the organization.

Although there was a generally positive attitude towards the perspectives and values that the model represents, the intention of the organization was rarely explicit regarding future implementation. Such ambivalent attitudes have been noted as a challenge in implementation and sustainability (Proctor et al., 2011). However, at the same time as lack of evidence and evaluations of peer support appeared to weaken the legitimacy of the model, the fact that the project was organized and supported by a user organization was noted to strengthen it. The access to experiential knowledge and competence, from the user movement as well as from the individual PSWs, was seen as valuable for service development. It is likely that PSW models not developed directly by a user organization should consider developing structural connections to these organization's and the knowledge base they represent as well.

These results, which point to the intersection of implementation, education, supervision and culture, suggest that the particular model studied here, with a user organization as facilitator, is particularly interesting. While contributing to some administrative and sustainability challenges, considering the user movement involvement as an essential component of an implementation team or process is worth 
further study. Can their active involvement as 'culture-bearers' be a key component in supporting the paradigm shift that managers note PSWs provoke, and is it important to have one foot in civil society in order to maintain the critical, rights-based perspective that even these managers noted the value of?

While collaboration with NSPH in this case was most often adequate, it was evident in one region that the dominant part played by NSPH as a national umbrella organization had contributed to a failure to involve the local user organizations in the project. According to implementation research, a high level of cosmopolitanism (Damschroder et al., 2009), or collaboration with 'external purveyor organizations' (Blum \& Roman, 1988; Fixsen et al., 2009) such as local user organizations, can create a more stable platform of the type this project sought to develop and facilitate a sustainable implementation.

The results suggest the specific, knowledge-based contributions made by NSPH may be understood as an implementation facilitator (Meyers et al., 2012) When, for example, managers highlighted the recruitment process recommended by NSPH, they commonly emphasized the importance of PSWs readiness in terms of having reached a stage in their recovery process that is commonly advised in international literature (Ibrahim et al., 2020). Managers also valued how this external, critical perspective could contribute to cultural change, recovery orientation and user participation in program development as well as in decision-making processes for individual service users. At the same time, uncertainty and sensitivity to such a 'critical outsider' perspective was often expressed.

For the most part, managers expressed approval of the levels of competency and skills of the PSWs, validating the quality of the comprehensive PSW training and supervision model developed by NSPH. Training is highlighted in the literature as a facilitator for peer support implementation (Ibrahim et al., 2020; Kent, 2019). While ongoing supervision was provided for the PSWs, managers and staff rarely received planned supervision from the user organization unless specifically requested. The question of ongoing supervision for staff in relation to the PSW perspective might also be linked in future studies to their need for user perspectives in their work as a whole.

Many of the implementation factors identified above are common to recent implementation studies on peer support (Ibrahim et al., 2020; Kent, 2019). In comparison with other interventions, the implementation of peer support seems to require a comprehensive shift in perspectives, power dynamics and culture. This is evident from descriptions of initial uncertainties in terms of fears, fantasies and prejudices. However, attitudes seem to shift rather easily, when participating in training and becoming acquainted with PSWs, to become overwhelmingly positive. The current findings, highlighting peer support as a catalyst for progress, corresponds with previous literature discerning how implementation of peer support might facilitate change in care-giving cultures (Rosenberg \& Argentzell, 2018).

While in the current study PSWs did not emerge as fully defined members of the work force, they had in most accounts, successfully left behind an identity as a primarily 'ill person'. They were described as unexpectedly surpassing the managers' expectations for being able to productively contribute to a recovery-oriented mental health system. At the same time, roles, tasks and their value as viable team 
members had not been established, an issue of particular concern for managers implementing this service. This liminal position, neither one or the other, suggests that PSWs, remain in a vulnerable position. Confusion and conflict within the role have frequently been highlighted as a barrier to PSW implementation (Asad \& Chreim, 2016; Farkas \& Boevink, 2018; Gates \& Akabas, 2007; Ibrahim et al., 2020; Kent, 2019; Mancini, 2018; Repper \& Carter, 2011; Vandewalle et al., 2016).

\section{Conclusions}

Overall, numerous positive experiences were noted from a managerial perspective from having a PSW in the workplace, both in respect to the working climate and the communication with service users. In implementation research, it is commonly highlighted how leadership motivation to implement a novel intervention is strengthened by learning of positive effects from decision makers in other services (Rogers, 2010). The current analysis highlighted how initial concerns amongst staff, in relation to the PSW role and responsibilities, proved unfounded, and might therefore, serve to ease managers' doubts in future projects.

Correspondingly, though the findings showed that implementation was rarely based on any needs assessment, (an organization level implementation component as specified in the SIS framework, table 2), it was evident how having a PSW in the team had often fulfilled and consequently defined needs that managers initially were not aware of. Most importantly, an organizational fit was noted in relation to team synergies, where the PSW improved the working climate and added value in client work. This finding responds to a knowledge gap highlighted in a recent review on PSWs' influence in work teams, identifying how reports are lacking on the contributions of PSWs' expertise in interprofessional learning (Viking et al., 2022).

The purpose of the NSPH project was to introduce PSWs as a new occupational category in a Swedish mental health context. However, questions remain regarding the nature of peer support that from a leadership perspective complicate implementation. While we have studied the implementation of peer support, using a tool which builds on implementation science, we should note that there is continuing uncertainty, as noted in previous research (Gillard et al., 2017; Rosenberg \& Argentzell, 2018), that peer support is a 'method' that can be implemented. It remains to be decided whether peer support is an intervention, a change process or a new profession, since these phenomena require different implementation strategies. The results of our study seem to confirm the uncertain nature of peer support, as many of the conclusions regarding role, identity, employer, connection to the user movement suggest, that there are still many questions as to what is being implemented. These are issues that middle and higher-level managers will have to address if these workers are to achieve a status as an integrated and sustainable support in mental health services.

\section{Study Limitations}


Working with a naturalistic approach brings with it a number of challenges as well. These include difficulties in creating continuity in the design, in the involvement of research personnel, and in responding to local needs at the same time as the broader study (Drill et al., 2019). In this case, these were complicated by a lack of centralized research funding, building instead on the willingness of local projects to fund and support follow-up studies and share data with the national project.

\section{References}

Aarons, G. A., Hurlburt, M., \& Horwitz, S. M. (2011). Advancing a conceptual model of evidence-based practice implementation in public service sectors. Administration and Policy in Mental Health and Mental Health Services Research, 38(1), 4-23.

Agrawal, S., Kalocsai, C., Capponi, P., Kidd, S., Ringsted, C., Wiljer, D., \& Soklaridis, S. (2021). "It was great to break down the walls between patient and provider": Liminality in a co-produced advisory course for psychiatry residents. Advances in Health Sciences Education, 26(2), 385-403.

Asad, S., \& Chreim, S. (2016). Peer support providers' role experiences on interprofessional mental health care teams: A qualitative study. Community Mental Health Journal, 52(7), 767-774. https://doi.org/https://doi.org/10.1007/s10597-015-9970-5

Bergmark, M., Bejerholm, U., \& Markström, U. (2019). Implementation of evidence-based interventions: Analyzing critical components for sustainability in community mental health services. Social Work in Mental Health, 17(2), 129-148. https://doi.org/https://doi.org/10.1080/15332985.2018.1511500

Blum, T. C., \& Roman, P. M. (1988). Purveyor organizations and the implementation of employee assistance programs. The Journal of Applied Behavioral Science, 24(4), 397-411.

https://doi.org/https://doi.org/10.1177/002188638802400407

Chinman, M., George, P., Dougherty, R. H., Daniels, A. S., Ghose, S. S., Swift, A., \& Delphin-Rittmon, M. E. (2014). Peer support services for individuals with serious mental illnesses: assessing the evidence. Psychiatric Services, 65(4), 429-441.

Crane, D. A., Lepicki, T., \& Knudsen, K. (2016). Unique and common elements of the role of peer support in the context of traditional mental health services. Psychiatric Rehabilitation Journal, 39(3), 282-288. https://doi.org/https://doi.org/10.1037/prj0000186

Czarniawska, B., \& Mazza, C. (2003). Consulting as a liminal space. Human Relations, 56(3), 267-290. https://doi.org/https://doi.org/10.1177/0018726703056003612

Damschroder, L. J., Aron, D. C., Keith, R. E., Kirsh, S. R., Alexander, J. A., \& Lowery, J. C. (2009). Fostering implementation of health services research findings into practice: A consolidated framework for advancing implementation science. Implementation Science, 4(1), 1-15.

https://doi.org/https://doi.org/10.1186/1748-5908-4-50 
Davidson, L., \& Guy, K. (2012). Peer support among persons with severe mental illnesses: A review of evidence and experience. World Psychiatry, 11(2), 123-128.

https://doi.org/https://doi.org/10.1016/j.wpsyc.2012.05.009

Drill, R., Drinkwater, R., Ernst, J., Gill, A., Hunnicutt, P., Mclntyre, S., Potter, C. M., Richardson, H., \& Beinashowitz, J. (2019). The vicissitudes of conducting psychodynamic research in a naturalistic setting. Psychotherapy, 56(1), 126-133. https://doi.org/https://doi.org/10.1037/pst0000181

Durlak, J. A., \& DuPre, E. P. (2008). Implementation matters: A review of research on the influence of implementation on program outcomes and the factors affecting implementation. American Journal of Community Psychology, 41(3), 327-350. https://doi.org/https://doi.org/10.1007/s10464-008-9165-0

Erlandson, D. A., Harris, E. L., Skipper, B. L., \& Allen, S. D. (1993). Doing naturalistic inquiry: A guide to methods. Sage.

Erlingsson, C., \& Brysiewicz, P. (2017). A hands-on guide to doing content analysis. African Journal of Emergency Medicine, 7(3), 93-99. https://doi.org/https://doi.org/10.1016/j.afjem.2017.08.001

Farkas, M., \& Boevink, W. (2018). Peer delivered services in mental health care in 2018: infancy or adolescence? World Psychiatry, 17(2), 222.

Fixsen, D. L., Blase, K. A., Naoom, S. F., \& Wallace, F. (2009). Core implementation components. Research on Social Work Practice, 19(5), 531-540. https://doi.org/https://doi.org/10.1177/1049731509335549

Fuhr, D. C., Salisbury, T. T., De Silva, M. J., Atif, N., van Ginneken, N., Rahman, A., \& Patel, V. (2014). Effectiveness of peer-delivered interventions for severe mental illness and depression on clinical and psychosocial outcomes: a systematic review and meta-analysis. Social Psychiatry and Psychiatric Epidemiology, 49(11), 1691-1702.

Gates, L. B., \& Akabas, S. H. (2007). Developing strategies to integrate peer providers into the staff of mental health agencies. Administration and Policy in Mental Health and Mental Health Services Research, 34(3), 293-306. https://doi.org/ https://doi.org/10.1007/s10488-006-0109-4

Gillard, S., Foster, R., Gibson, S., Goldsmith, L., Marks, J., \& White, S. (2017). Describing a principles-based approach to developing and evaluating peer worker roles as peer support moves into mainstream mental health services. Mental Health and Social Inclusion, 12(3), 133-143.

https://doi.org/https://doi.org/10.1108/mhsi-03-2017-0016

Graneheim, U. H., Lindgren, B.-M., \& Lundman, B. (2017). Methodological challenges in qualitative content analysis: A discussion paper. Nurse Education Today, 56, 29-34.

https://doi.org/https://doi.org/10.1016/j.nedt.2017.06.002

Hollweck, T. (2015). Robert K. Yin.(2014). Case Study Research Design and Methods . Thousand Oaks, CA: Sage. 282 pages. Canadian Journal of Program Evaluation, 30(1), 108-110. 
Hsieh, H.-F., \& Shannon, S. E. (2005). Three approaches to qualitative content analysis. Qualitative Health Research, 15(9), 1277-1288. https://doi.org/https://doi.org/10.1177/1049732305276687

Ibrahim, N., Thompson, D., Nixdorf, R., Kalha, J., Mpango, R., Moran, G., Mueller-Stierlin, A., Ryan, G., Mahlke, C., \& Shamba, D. (2020). A systematic review of influences on implementation of peer support work for adults with mental health problems. Social Psychiatry and Psychiatric Epidemiology, 55(3), 285293. https://doi.org/ https://doi.org/10.1007/s00127-019-01739-1

Kemp, V., \& Henderson, A. R. (2012). Challenges faced by mental health peer support workers: Peer support from the peer supporter's point of view. Psychiatric Rehabilitation Journal, 35(4), 337-340. https://doi.org/ https://doi.org/10.2975/35.4.2012.337.340

Kent, M. (2019). Developing a strategy to embed peer support into mental health systems. Administration and Policy in Mental Health and Mental Health Services Research, 46(3), 271-276.

https://doi.org/https://doi.org/10.1007/s10488-018-0912-8

Mancini, M. A. (2018). An exploration of factors that effect the implementation of peer support services in community mental health settings. Community Mental Health Journal, 54(2), 127-137.

https://doi.org/https://doi.org/10.1007/s10597-017-0145-4

Markström, U., Svensson, B., Bergmark, M., Hansson, L., \& Bejerholm, U. (2018). What influences a sustainable implementation of evidence-based interventions in community mental health services? Development and pilot testing of a tool for mapping core components. Journal of Mental Health, 27(5), 395-401. https://doi.org/https://doi.org/10.1080/09638237.2017.1417544

Meyers, D. C., Durlak, J. A., \& Wandersman, A. (2012). The quality implementation framework: A synthesis of critical steps in the implementation process. American Journal of Community Psychology, 50(3-4), 462-480. https://doi.org/https://doi.org/10.1007/s10464-012-9522-x

Mutschler, C., Bellamy, C., Davidson, L., Lichtenstein, S., \& Kidd, S. (2021). Implementation of peer support in mental health services: A systematic review of the literature. Psychological Services.

Nordén, H., \& Falkman, M. (Eds.). (2018). Reportageboken: Peer support inom psykiatri och socialpsykiatri [The reportage book: Peer support in psychiatry and social psychiatry]. NSPHiG. https://nsphig.se/wpcontent/uploads/2019/03/reportagebok_dec11.pdf.

Proctor, E., Silmere, H., Raghavan, R., Hovmand, P., Aarons, G., Bunger, A., Griffey, R., \& Hensley, M. (2011). Outcomes for implementation research: Conceptual distinctions, measurement challenges, and research agenda. Administration and Policy in Mental Health and Mental Health Services Research, 38(2), 65-76. https://doi.org/ https://doi.org/10.1007/s10488-010-0319-7

Repper, J., Aldridge, B., Gilfoyle, S., Gillard, S., Perkins, R., \& Rennison, J. (2013). Peer support workers: Theory and practice. ImROC. https://imroc.org/wp-content/uploads/2013/06/5ImROC-Peer-Support- 
Workers-Theory-and-Practice.pdf

Repper, J., \& Carter, T. (2011). A review of the literature on peer support in mental health services. Journal of Mental Health, 20(4), 392-411. https://doi.org/https://doi.org/10.2196/preprints.15572

Rogers, E. M. (2010). Diffusion of innovations. Simon and Schuster.

Rogers, E. S., Maru, M., Johnson, G., Cohee, J., Hinkel, J., \& Hashemi, L. (2016). A randomized trial of individual peer support for adults with psychiatric disabilities undergoing civil commitment. Psychiatric Rehabilitation Journal, 39(3), 248-255. https://doi.org/https://doi.org/10.1037/prj0000208

Rosenberg, D., \& Argentzell, E. (2018). Service users experience of peer support in Swedish mental health care: A "tipping point" in the care-giving culture? Journal of Psychosocial Rehabilitation and Mental Health, 5(1), 53-61. https://doi.org/https://doi.org/10.1007/s40737-018-0109-1

Rubin, R. M., Hurford, M. O., Hadley, T., Matlin, S., Weaver, S., \& Evans, A. C. (2016). Synchronizing watches: The challenge of aligning implementation science and public systems. Administration and Policy in Mental Health and Mental Health Services Research, 43(6), 1023-1028.

https://doi.org/https://doi.org/10.1007/s10488-016-0759-9

Salzer, M. S., Rogers, J., Salandra, N., O'Callaghan, C., Fulton, F., Balletta, A. A., Pizziketti, K., \& Brusilovskiy, E. (2016). Effectiveness of peer-delivered Center for Independent Living supports for individuals with psychiatric disabilities: A randomized, controlled trial. Psychiatric Rehabilitation Journal, 39(3), 239-247. https://doi.org/https://doi.org/10.1037/prj0000220

Silver, J., \& Nemec, P. B. (2016). The role of the peer specialists: Unanswered questions. Psychiatric Rehabilitation Journal, 39(3), 289-291. https://doi.org/https://doi.org/10.1037/prj0000216

Slade, M., Amering, M., Farkas, M., Hamilton, B., O'Hagan, M., Panther, G., Perkins, R., Shepherd, G., Tse, S., \& Whitley, R. (2014). Uses and abuses of recovery: Implementing recovery-oriented practices in mental health systems. World Psychiatry, 13(1), 12-20. https://doi.org/https://doi.org/10.1002/wps.20084

Slade, M., Oades, L., \& Jarden, A. (2017). Wellbeing, recovery and mental health. Cambridge University Press.

Socialstyrelsen [National Board of Health and Welfare]. (2017). Vård och stöd vid schizofreni och schizofreniliknande tillstånd: Stöd för styrning och ledning [Care and support for schizophrenia and schizophrenia like conditions: Support for guidance and management]. Socialstyrelsen.

Stake, R. E. (1995). The art of case study research. Sage.

Vandewalle, J., Debyser, B., Beeckman, D., Vandecasteele, T., Van Hecke, A., \& Verhaeghe, S. (2016). Peer workers' perceptions and experiences of barriers to implementation of peer worker roles in mental health 
services: A literature review. International Journal of Nursing Studies, 60, 234-250.

https://doi.org/https://doi.org/10.1016/j.ijnurstu.2016.04.018

Viking, T., Wenzer, J., Hylin, U., \& Nilsson, L. (2022). Peer support workers' role and expertise and interprofessional learning in mental health care: A scoping review. Journal of Interprofessional Care, 1-11. https://doi.org/10.1080/13561820.2021.2014796

\section{Declarations}

\section{Ethics approval and consent to participate}

The study was approved by the Regional Ethics Review Board in Lund, Sweden (Dnr 2015:218). Informed consent was obtained and all managers were informed that they could withdraw their consent to participate at any time.

\section{Consent for publication}

All respondents provided written informed consent for publication. All authors read and approved the final manuscript.

\section{Availability of data and materials}

Data will not be readily available because they consist of interview data, for which confidentiality cannot be ensured. Requests to access the datasets should be directed to KG.

\section{Competing interests}

The authors declare that they have no competing interests.

\section{Funding}

The authors have not received any funding or benefits from industry or elsewhere to conduct this study.

\section{Authors' contributions}

DR and EA managed the research project and designed the study. Moreover, they trained the local interviewers and supervised the interviews. MB belong to the group of researchers who have developed the implementation framework employed in the various stages of research and contributed with his expertise in the research field of implementation. KG had the main responsibility in analyzing the data 
and writing up the manuscript. However, data analysis and writing has been performed in close collaboration with all four authors.

\section{Acknowledgements}

We thank our respondents who shared their experiences and views and made this study possible.

\section{Tables}

Table 1. Included workplaces

\begin{tabular}{|c|c|c|c|}
\hline Region & Included managers $(\mathrm{N})$ & Settings of services & \\
\hline & Total $(\mathrm{N}=18)$ & $\begin{array}{l}\text { County based psychiatry } \\
\text { regional services }\end{array}$ & $\begin{array}{r}\text { Social psychiatric } \\
\text { municipal services }\end{array}$ \\
\hline A & $\begin{array}{l}\text { Managers }(n=7) \\
\text { Services }(n=6) \\
\text { (Two respondents belonged to the same } \\
\text { service) }\end{array}$ & 0 & 7 \\
\hline B & $\begin{array}{l}\text { Managers }(n=5) \\
\text { Services }(n=4) \\
\text { (One of the managers worked on a } \\
\text { central-) and not unit-level }\end{array}$ & 5 & 0 \\
\hline $\mathrm{C}$ & $\begin{array}{l}\text { Managers }(n=6) \\
\text { Services }(n=6)\end{array}$ & 3 & 3 \\
\hline
\end{tabular}

In Region A and B, Recruitment and employment of PSWs was arranged by NSPH. Region C had established systematic forms for user involvement within their organizations. Recruitment and employment were provided by local actors, centrally embedded in the organization.

Table 2. SIS components (Sustainable implementation scale) 


\begin{tabular}{lll}
\hline Organizational Level & Team Level & Continuous Support \\
\hline Assessment of needs for the model & Selection of staff & Continuing training \\
Experiences of the model & Continuity among staff & Supervision \\
The model's legitimacy & Available leader & Recurrent fidelity assessments \\
Organizational fit & Collaboration partner & Time for reflection \\
Implementation climate & Information strategies & Administrative support \\
Collaboration culture & Feedback to financiers & \\
Leaders engagement & Training & \\
Local champions & & \\
External experts & & \\
Financial strategy & & \\
Steering group & & \\
Collaboration partners & & \\
\hline
\end{tabular}

\section{Table 3. Theme, main categories and categories}

Navigating liminal spaces when implementing PSW in mental health systems

\begin{tabular}{|c|c|c|c|c|c|c|c|}
\hline \multicolumn{2}{|c|}{ centives and conditions } & \multicolumn{3}{|c|}{ Implementation and organization } & \multicolumn{3}{|c|}{ Experiences of peer support } \\
\hline $\begin{array}{l}\text { Opportunities } \\
\text { for full scale } \\
\text { support }\end{array}$ & $\begin{array}{c}\text { User } \\
\text { movement } \\
\text { collaboration }\end{array}$ & $\begin{array}{c}\text { Preparation } \\
\text { phase at } \\
\text { the start }\end{array}$ & $\begin{array}{c}\text { The } \\
\text { working } \\
\text { role of the } \\
\text { PSW }\end{array}$ & $\begin{array}{c}\text { Continuous } \\
\text { Support }\end{array}$ & $\begin{array}{l}\text { The } \\
\text { PSW in } \\
\text { the } \\
\text { client } \\
\text { work }\end{array}$ & $\begin{array}{l}\text { The PSW } \\
\text { in the } \\
\text { working } \\
\text { team }\end{array}$ & $\begin{array}{l}\text { Thoughts } \\
\text { on the } \\
\text { future }\end{array}$ \\
\hline
\end{tabular}

